\title{
La Heterogeneidad de los Servicios Intensivos en Conocimiento: El Caso de Argentina ${ }^{* *}$
}

\author{
Andrés López $z^{1}$ Andrés Niembro ${ }^{2 *}$
}

Resumen: El presente artículo busca contribuir al debate acerca de la heterogeneidad entre los servicios intensivos en conocimiento (SIC), sobre la base de una fuente de información novedosa que nos permite no sólo estudiar las características de los SIC a un mayor nivel de desagregación que el utilizado comúnmente en la literatura internacional, sino también analizar estas actividades en el marco de un país en desarrollo como la Argentina. Mediante una combinación de técnicas de análisis multivariado obtenemos una tipología empírica que nos brinda una visión más clara sobre los puntos en común y las diferencias entre los distintos tipos de SIC identificados. Esta contribución no sólo es útil para el mejor análisis de estos sectores, que han ganado fuerte peso en la economía global, sino también para los tomadores de decisión, ya que son muchos los países que están implementando políticas de desarrollo productivo dirigidas a los SIC.

Palabras clave: servicios intensivos en conocimiento; heterogeneidad; tipología; países en desarrollo; análisis multivariado

\section{The heterogeneity of knowledge-intensive services: the case of Argentina}

Abstract: The present paper seeks to contribute to the debate about the heterogeneity between knowledge-intensive services (KIS), based on a novel source of information that allows us not only to study the characteristics of KIS with a higher level of disaggregation than most of the international literature, but also to analyze these activities from the point of view of a developing country such as Argentina. Through a combination of multivariate analysis techniques we finally obtain an empirical typology that gives us a clearer picture about the similarities and differences among different types of KIS. Conclusions are relevant not only for the academic analysis of these sectors, which are increasingly relevant in the world economy, but also for policy makers, since many countries are implementing industrial policies aimed at promoting KIS.

Keywords: knowledge-intensive services; heterogeneity; typology; developing countries; multivariate analysis

Submitted: March $5^{\text {th }}, 2018 /$ Approved: November $6^{\text {th }}, 2019$

\section{Introducción}

A partir de la clasificación residual (o complementaria) adoptada varias décadas atrás por Fisher (1939) y Clark (1940), dentro del sector terciario o de servicios se incluyeron todos aquellos rubros que no podían ubicarse entre las actividades primarias o en las secundariasmanufactureras. Así, bajo el paraguas de los servicios se han englobado una gran variedad de actividades heterogéneas entre sí (Bastos y Perobelli, 2012; Glückler y Hammer, 2011; 2013). En años más recientes, como señalan Becker, Böttcher y Klingner (2011), debido a la toma de conciencia de esta heterogeneidad y también como una forma de manejarla, se han desarrollado diferentes esfuerzos por desagregar y/o re-clasificar a los servicios.

Por un lado, es posible encontrar distintas clasificaciones oficiales de actividades económicas, diseñadas y aplicadas por organismos estadísticos para estructurar la recopilación de información y facilitar el análisis de la misma. Por otra parte, como comenta Viitamo (2007), también se han derivado tipologías, tanto teóricas como empíricas, con el fin de resaltar ciertas características o dimensiones que en ocasiones pueden verse obscurecidas por las clasificaciones oficiales y que serían comunes a distintos subconjuntos o clusters de actividades de servicios.

No obstante, las tipologías teóricas resultan en cierta medida incompletas o parciales, ya que se basan en definiciones idiosincráticas y sus resultados dependen considerablemente de los criterios adoptados (Glückler y Hammer, 2011; 2013; Lee, Shim, Jeong y Hwang, 2003). En tanto, un problema asociado a muchas de las tipologías empíricas es que el análisis se suele realizar a partir de datos sectoriales a uno o dos dígitos de desagregación, impidiendo identificar las heterogeneidades hacia el interior de estos agregados.

Dentro de los servicios, un subconjunto particular que ha ganado crecientemente atención e importancia en las últimas décadas, pero que no se encuentra por ello al margen de las consideraciones generales anteriores, es el de los servicios intensivos en conocimiento (SIC). Este grupo de actividades, cuyo análisis remonta al aporte seminal de Miles et al. (1995), atrajo rápidamente el interés de investigadores y

** Versiones previas de este trabajo fueron presentadas durante 2017 en la VI Conferencia Internacional de REDLAS (San José, Costa Rica) y en la LII Reunión Anual de la Asociación Argentina de Economía Política (Bariloche, Argentina). Los autores agradecen los valiosos comentarios recibidos en ambos eventos como así también la gestión de la Secretaria de Transformación Productiva del Ministerio de Producción de la República Argentina para la obtención de los datos en los que se basa el presente artículo. Como es usual, los errores remanentes son de nuestra exclusiva responsabilidad.

1) Instituto Interdisciplinario de Economía Política de Buenos Aires (IIEP-BAIRES), Universidad de Buenos Aires y Consejo Nacional de Investigaciones Científicas y Técnicas (CONICET), Ciudad Autónoma de Buenos Aires, Argentina.

2) Centro Interdisciplinario de Estudios sobre Territorio, Economía y Sociedad (CIETES), Universidad Nacional de Río Negro, Río Negro, Argentina.

*Autor de correspondencia: aniembro@unrn.edu.ar 
hacedores de política, ya que se trata de servicios de un elevado valor agregado, que generan puestos de trabajo de relativamente alta calificación y son una pieza fundamental en la economía del conocimiento, tanto por su propia inclinación hacia la innovación como por su rol en materia de transmisión de conocimiento y facilitación de las actividades innovativas en otros sectores (Den Hertog, 2000; Freel, 2006; Glückler y Hammer, 2011; Howells, 2000; Muller y Zenker, 2001; Schnabl y Zenker, 2013; Tether y Hipp, 2002).

En América Latina estos sectores también han tenido un desarrollo importante en los últimos años, aumentando incluso su inserción en los mercados externos, tal como se analizó en López, Niembro y Ramos (2014). En el caso de Argentina, según cálculos recientes de López (2018), los SIC han incrementado su participación tanto en materia de empleo como de valor agregado (lo cual está en línea con lo observado en los Estados Unidos y la Unión Europea), a la vez que ha crecido su vocación exportadora. Asimismo, cabe destacar que las empresas argentinas de SIC resultan más grandes que el promedio de la economía si se las mide por número de empleados y suelen pagar salarios también por encima de la media general. Esto se suma al hecho de que, en comparación con otros sectores, los sueldos y contribuciones sociales representan una parte considerable de los costos, todo lo cual refleja el carácter trabajo-intensivo de estas actividades (López, 2018). Sin embargo, como veremos luego, estas características generales pueden ocultar importantes diferencias entre los subsectores de SIC.

En algunos trabajos previos parece adoptarse la postura de que los servicios intensivos en conocimiento serían un conjunto de actividades relativamente homogéneas entre sí (Den Hertog y Bilderbeek, 2000; Tether, 2005). De hecho, varios estudios empíricos de países desarrollados y también de América Latina tienden a concentrarse únicamente en enfatizar las diferencias entre los SIC y el resto de las actividades de servicios (Aboal y Garda, 2016; Álvarez, Bravo-Ortega y Zahler, 2015; Asikainen, 2015; Crespi, Tacsir y Vargas, 2014; Dutrénit, De Fuentes, Santiago, Torres y Gras, 2013; Gallego, Gutiérrez y Taborda, 2015; Hollenstein, 2003; Tello, 2017; Tether, 2005; Torrecillas y Brandão Fischer, 2011). No obstante, hay quienes sostienen que en realidad existe una marcada heterogeneidad hacia el interior de los SIC (Consoli y Elche-Hortelano, 2010; Corrocher, Cusmano y Morrison, 2009; Doloreux, Amara y Landry, 2008). Por otra parte, varios de los trabajos sobre SIC se han basado en categorías (teóricas) muy agregadas, como por ejemplo la distinción de Miles et al. (1995) entre SIC tecnológicos y profesionales (Consoli y Elche-Hortelano, 2010; Corrocher et al., 2009; Freel, 2006; Muller y Zenker, 2001; Zenker y Doloreux, 2008), por lo que resulta necesario bajar un poco más para examinar las diferencias entre los sectores agrupados dentro de estos agregados. Sin embargo, la limitada disponibilidad de datos más allá de los dos dígitos en las distintas clasificaciones de actividades económicas ha restringido considerablemente la profundidad de estos esfuerzos (Doloreux et al., 2008; Schnabl y Zenker, 2013).

En este artículo, en cambio, la base de datos que disponemos nos permite trabajar a tres dígitos y así poder ser más precisos en la identificación de similitudes y diferencias entre las actividades que se incluyen dentro del universo de los SIC. Asimismo, en contraposición a otros trabajos que se basan en la validación empírica de tipologías conceptuales derivadas previamente (David, 2014; Freel, 2006; Hipp y Grupp, 2005; Verma y Boyer, 2000), buscamos analizar la heterogeneidad de los SIC a partir de los propios datos a nivel de sectores y extraer de este modo una tipología empírica. En este sentido, compartimos el punto de vista de Corrocher et al. (2009) de que es necesario explorar la caja negra de los SIC y que, para ello, hay que buscar patrones en los datos, dejando de lado los agregados sectoriales definidos por clasificaciones a priori, que pueden ocultar características posiblemente relevantes para diferenciar los sectores entre sí.

Dicho todo lo anterior, este artículo pretende contribuir al debate sobre la heterogeneidad hacia el interior de los SIC sobre la base de una fuente de información novedosa que permite, por un lado, estudiar las características de estas actividades económicas a tres dígitos de desagregación y, por otro, analizar el caso de un país en desarrollo de América Latina como la Argentina. Mediante una combinación de técnicas de análisis multivariado, se obtiene finalmente una tipología empírica que brinda una visión más clara y concreta sobre los puntos en común y las diferencias entre los distintos tipos de SIC.

El artículo se estructura de la siguiente forma. En la segunda sección se realiza una breve reseña de la literatura que antecede al presente trabajo. Luego, en la tercera sección se describen las fuentes de información y los datos utilizados, como así también las técnicas multivariantes y los criterios metodológicos adoptados. La cuarta sección reúne los resultados obtenidos en las distintas etapas del estudio: por un lado, los componentes principales y las dimensiones de análisis; y por otro, los clusters y distintos tipos de SIC identificados. Por último, se concluye con algunas reflexiones finales a partir de dicho ejercicio.

\section{Marco conceptual y antecedentes}

A lo largo del tiempo se ha ido gestando una importante cantidad de esfuerzos de desagregación, clasificación o desarrollo de tipologías para el sector de servicios (Becker et al., 2011; Browning y Singelmann, 1975; Cook, Goh y Chung, 1999; Liu, Wang y Lee, 2008; Lovelock, 1983; Meiren et al., 2015; Shafti, Van Der Meer y Williams, 2007; Silvestro, Fitzgerald, Johnston y Voss, 1992; Stigler, 1956). Por dar algunos ejemplos, Becker et al. (2011) identifican entre 1923 y 2011 un total de 81 sistemas de clasificación de servicios, mientras que Meiren et al. (2015) encuentran que se han utilizado más de 50 criterios diferentes para tal propósito.

Dentro de esta gran diversidad, y teniendo en cuenta el objetivo del presente artículo, vale destacar algunos trabajos que han recurrido a técnicas de análisis multivariante para contrastar empíricamente tipologías conceptuales o bien para derivar tipologías empíricas a partir de los datos y sin adoptar una taxonomía teórica a priori. En el primero de los casos se ubica el aporte de Verma y Boyer (2000), quienes emplean el análisis factorial para testear y validar empíricamente una tipología desarrollada previamente por Schmenner (1986). Por su parte, David (2014) recurre a datos económicos y al análisis de componentes principales para recrear, triangular y validar la clasificación de servicios realizada previamente por Shafti et al. (2007). 
Dentro del segundo grupo se encuentra el trabajo de Meiren et al. (2015), quienes derivan empíricamente una tipología de servicios a partir de una base de datos de empresas de diferentes sectores y países, utilizando tanto el análisis factorial, para reducir el número de dimensiones de comparación, como así también el análisis cluster, para armar grupos de empresas con características similares entre sí y, a su vez, diferentes de las cualidades de otros conjuntos.

Con respecto a los sectores de nuestro interés, y siguiendo la definición seminal de Miles et al. (1995), los servicios intensivos en conocimiento abarcan a todas aquellas actividades de servicios que impactan en la creación, acumulación o difusión de conocimiento. De modo similar, Den Hertog (2000) señala que los SIC funcionan tanto como fuente de innovaciones así también como facilitadores o transmisores de las mismas y que se requiere de un fuerte componente de conocimiento profesional o experiencia en disciplinas técnicas específicas para poder proveer este tipo de servicios. Rubalcaba, Gallego y Den Hertog (2010) también destacan que, a diferencia de los servicios más tradicionales, los SIC se caracterizan por el papel central que juega el conocimiento acumulado en el personal y las rutinas organizacionales. Es por esto que tanto los inputs como los outputs de los SIC incluyen un alto grado de conocimiento tácito (Doloreux et al., 2008). En palabras de Hipp y Grupp (2005), los SIC tienden a combinar conocimientos de diferentes fuentes y luego distribuir nuevo conocimiento hacia el resto de la economía, mientras que Schnabl y Zenker (2013) sostienen que las firmas de estos sectores se suelen ocupar de la recolección, procesamiento y tratamiento del conocimiento y de su adaptación a las necesidades de los clientes. En definitiva, los SIC comparten la característica de emplear capital humano de relativamente alto nivel de calificación y de ser usuarios y, en general también, productores de información y conocimiento.

Respecto al estudio de los SIC, en primera instancia surgieron una serie de tipologías teóricas derivadas de modelos conceptuales o bien de matrices donde se entrecruzaban distintas características de dichas actividades. Un ejemplo es la taxonomía de innovación en servicios presentada por Miozzo y Soete (2001), la cual se inspiraba y aportaba una extensión a la contribución original de Pavitt (1984). Por otra parte, en el trabajo seminal de Miles et al. (1995: 29-30) se diferencian los "nuevos servicios intensivos en tecnología" (T-SIC), mayormente relacionados con la oferta vinculada a las TICs y ciertas actividades técnicas, de los "servicios profesionales tradicionales" (P-SIC), eventualmente usuarios de las nuevas tecnologías (que, entre otras cosas, habilitan el surgimiento también de nuevas líneas de negocios).

Sobre esta base, varios trabajos apuntaron a contrastar empíricamente dichas tipologías teóricas. Por ejemplo, Hipp y Grupp (2005) testean la taxonomía de Miozzo y Soete con datos provenientes de Alemania, mientras que otros hacen lo propio a partir de la distinción entre TSIC y P-SIC (Consoli y Elche-Hortelano, 2010; Corrocher et al., 2009; Doloreux et al., 2008; Freel, 2006; Muller y Zenker, 2001; Zenker y Doloreux, 2008). En general, se suele corroborar la existencia de diferentes patrones de comportamiento y evolución entre estos grupos de servicios, pero sin ahondar hacia el interior de los distintos sectores que los componen.
Otros autores han optado por desarrollar sus propias tipologías teóricas y luego validarlas empíricamente. Este es el caso, por ejemplo, de Von Nordenflycht (2010), quien sugiere la existencia de cuatro tipos diferentes de empresas de servicios (profesionales) intensivos en conocimiento e intenta ilustrar la utilidad de dicha tipología a partir de datos de los Estados Unidos. Por su parte, Glückler y Hammer $(2011 ; 2013)$ cruzan de forma conceptual tres dimensiones de análisis (orientación de la demanda, intensidad de conocimiento e intensidad tecnológica) e identifican cinco tipos diferentes de servicios, aunque sólo tres de estos corresponden a variantes de SIC (para el consumidor, empresariales y tecnológicos). En base a datos de Alemania a tres dígitos (según la clasificación europea NACE), clasifican 93 sectores entre estas cinco categorías y verifican la capacidad de dicha tipología para dar cuenta de la heterogeneidad sectorial y regional en el desarrollo de estas ramas de actividad.

En un aporte que guarda cierto vínculo con el presente artículo, Chica Mejía (2011) parte de la clasificación de sectores intensivos en conocimiento de la OCDE (que incluye tanto manufacturas como servicios) y realiza un análisis de componentes principales seguido por un análisis cluster para identificar conjuntos de sectores relativamente homogéneos en materia de calificación y formación de sus recursos humanos. De esta forma, verifica que existe una relación entre los grupos que reúnen a las ocupaciones más calificadas y los sectores que la OCDE clasifica como intensivos en conocimiento y tecnología.

Por otro lado, una orientación particular de la literatura de servicios ha sido el estudio (llevado a cabo mayormente de forma descriptiva) de los diferentes tipos de patrones de innovación que pueden encontrarse entre las actividades de servicios en general o específicamente de los SIC, enfatizándose la existencia de una marcada heterogeneidad inter-sectorial o entre grupos de sectores (Consoli y Elche-Hortelano, 2010; Doloreux et al., 2008; Freel, 2006; Lee et al., 2003; Tether, 2003; Tether y Hipp, 2002; Tödtling, Lehner y Trippl, 2006; Wong y He, 2005). A su vez, algunos trabajos en esta línea han recurrido al uso de técnicas de análisis multivariado, combinando usualmente el análisis factorial y el análisis cluster para comparar e identificar diferencias entre sectores y, eventualmente, proponer una tipología empírica sobre esta base (Asikainen, 2015; Corrocher et al., 2009; Evangelista, 2000).

Otra rama cercana de la literatura empírica se ha ocupado de clasificar las estrategias o patrones de innovación de las empresas de servicios (no de los sectores) y, a su vez, varios de estos aportes han apelado metodológicamente a la técnica de análisis cluster para el desarrollo de dichas tipologías (De Jong y Marsili, 2006; Elche Hortelano y Gongález-Moreno, 2007; Hollenstein, 2003; Jordá Borrell, 2007). En general, de estos trabajos sobresalen tanto la heterogeneidad hacia el interior de las firmas de servicios como también cierta diferenciación con las categorías previamente desarrolladas para sectores manufactureros.

Por último, vale destacar que la mayor parte de los trabajos empíricos mencionados se basan en evidencia recolectada en países desarrollados, con la excepción de la inclusión de México en Meiren et al. (2015). En el caso específico de América Latina, una serie de aportes relativamente recientes han buscado analizar los patrones de innovación en servicios en algunos países de la región, pero distinguiendo 
únicamente entre el conjunto de los SIC y el resto de los servicios tradicionales (Aboal y Garda, 2016; Álvarez et al., 2015; Crespi et al., 2014; Dutrénit et al., 2013; Gallego et al., 2015; Tello, 2017). A su vez, en la mayoría de estos trabajos la categoría de SIC se establece agregando sectores a 2 dígitos o incluso a 1 dígito, como en los casos de Chile y Perú (Álvarez et al., 2015; Tello, 2017). Por otro lado, y en línea con la metodología adoptada en este artículo (que desarrollaremos a continuación), Bastos y Perobelli (2012) recurren al análisis factorial para clasificar todos los sectores de servicios en Brasil (comprobando la heterogeneidad entre los mismos) y Borrastero (2014) utiliza el análisis factorial y cluster para agrupar a las empresas innovadoras en el sector de software de Argentina según el acceso a las políticas públicas nacionales.

\section{Datos y metodología}

Aunque en la literatura se han propuesto definiciones distintas sobre el concepto de servicios intensivos en conocimiento (Den Hertog, 2000; García-Quevedo, Mas-Verdú y Montolio, 2013; Wood, 2002), en la práctica hay cierto consenso sobre cuáles son los sectores que pertenecen al universo de los SIC, basado en gran medida en una serie de ejercicios de identificación y clasificación realizados en Europa y que ya se han establecido como una norma estándar a nivel internacional
(Doloreux et al., 2008; García Manjón, 2008; Muller y Doloreux, 2009; Schnabl y Zenker, 2013; Torrecillas y Brandão Fischer, 2011). De igual manera, en este trabajo se toma como punto de partida el conjunto de sectores de SIC propuesto por Eurostat (cuadro 1), el cual apunta a dar una cobertura relativamente amplia de este tipo de servicios.

No obstante, algunos de los sectores que Eurostat considera dentro de los SIC no podrán ser analizados en este trabajo (y se encuentran marcados en gris en el cuadro 1). Esto se debe a que nuestra principal fuente de información consiste en datos tributarios suministrados por la Administración Federal de Ingresos Públicos (AFIP), correspondientes a las presentaciones realizadas por las empresas del Impuesto al Valor Agregado (IVA), Seguridad Social y Ganancias de las sociedades. Si bien se trata de una fuente novedosa de datos para este tipo de ejercicios, y al igual que Stigler (1956) consideramos que los impuestos recaudados a nivel nacional representan una de las bases de información más detalladas, existen algunas limitaciones. La principal es que hay sectores, como los de la administración pública, educación, salud y servicios sociales, que no se encuentran suficientemente cubiertos con este tipo de impuestos, ya que gran parte de su provisión corresponde a organismos públicos o bien, por su fin social, las entidades privadas pueden acceder a distintos tipos de exenciones.

Cuadro 1. Actividades incluidas en la definición de SIC de Eurostat (NACE Rev.2 a 2 dígitos). Traducción propia de la clasificación de Eurostat.

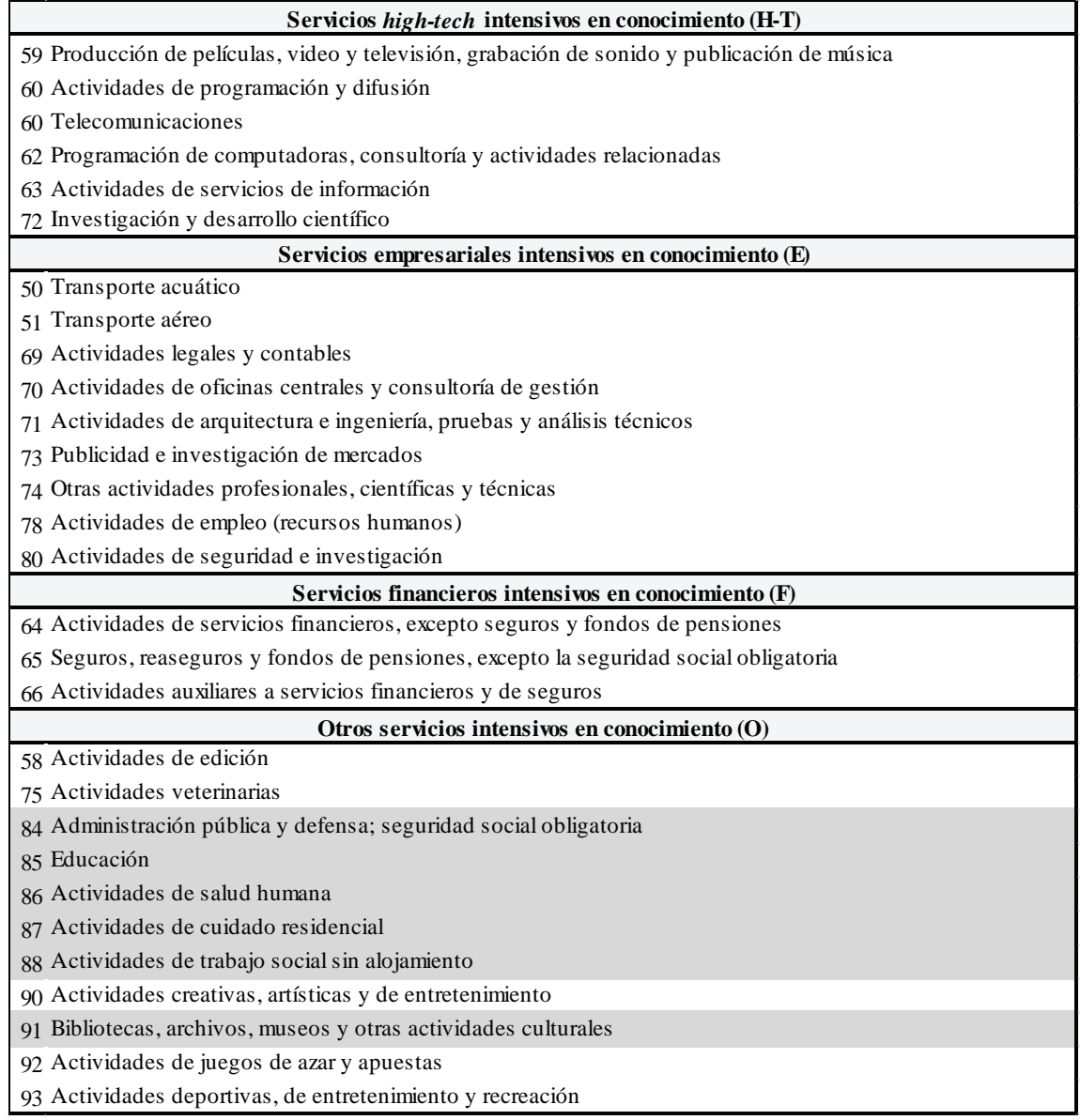

${ }^{1}$ Ver: http://ec.europa.eu/eurostat/statistics-explained/index.php/Glossary:Knowledge-intensive_services_(KIS) 
Por otro lado, en este trabajo optamos por incorporar algunos subsectores que Eurostat no considera dentro de su clasificación de SIC, pero que pueden ser de relevancia para el contexto de países en desarrollo como la Argentina. Como señalan Muller y Doloreux (2009), aunque algunos servicios especializados ligados a la explotación de recursos naturales no suelen incluirse entre los servicios intensivos en conocimiento, se puede tratar de actividades con características similares a estos, por ejemplo, en cuanto a los niveles de calificación del personal y el uso de nuevas tecnologías. De este modo, incluimos dentro de nuestra clasificación de SIC algunos servicios de apoyo a la actividad primaria, como así también a la gestión y logística y otros servicios empresariales no tenidos en cuenta en el caso europeo, pero que sí son de interés para países como los latinoamericanos (ver cuadro 2, donde estos sectores se resaltan en gris).

Una última diferencia con la clasificación de Eurostat, que responde al señalado interés por utilizar categorías menos agregadas, es que trabajamos con datos a 3 dígitos en lugar de 2. Para ello, hemos tenido que recortar nuestro horizonte de análisis a la información tributaria de 2014, ya que sólo para este último año los datos de AFIP disponibles se presentan a un mayor nivel de desagregación.
Otra particularidad de nuestra fuente de información es que los datos suministrados se encuentran agregados a nivel sectorial. El hecho de trabajar con promedios sectoriales impide analizar la heterogeneidad interna a cada actividad, ya que no disponemos de información acerca de la distribución de las firmas en torno a dichos promedios. Quedará para futuros trabajos avanzar en esa dirección en la medida en que se pueda acceder a información a nivel de empresa.

Más allá de los tradicionales límites que imponen la existencia de una economía informal y/o la subdeclaración de ingresos sobre la fiabilidad de los datos impositivos, también hay algunos otros puntos a tener presentes. Por ejemplo, cuando la información proviene de declaraciones juradas en donde las empresas informan su desempeño en las distintas variables de interés para su principal rama de actividad, puede perderse la información de aquellas firmas que también proveen SIC pese a que estos no son su negocio central (por ejemplo, una empresa industrial multinacional que tiene un centro de servicios compartidos que abastece a otras filiales de la corporación). Por otro lado, en muchos sectores podrían convivir empresas que efectivamente prestan SIC con otras que proveen servicios menos sofisticados. Asimismo, esta fuente de datos no incluye, por su propia naturaleza, ciertas variables que serían valiosas para una tipología de SIC (teniendo en cuenta la literatura internacional), como por ejemplo las vinculadas a actividades innovativas. 
Cuadro 2. Actividades incluidas en nuestra definición de SIC (CLAE-AFIP a 3 dígitos) ${ }^{1}$

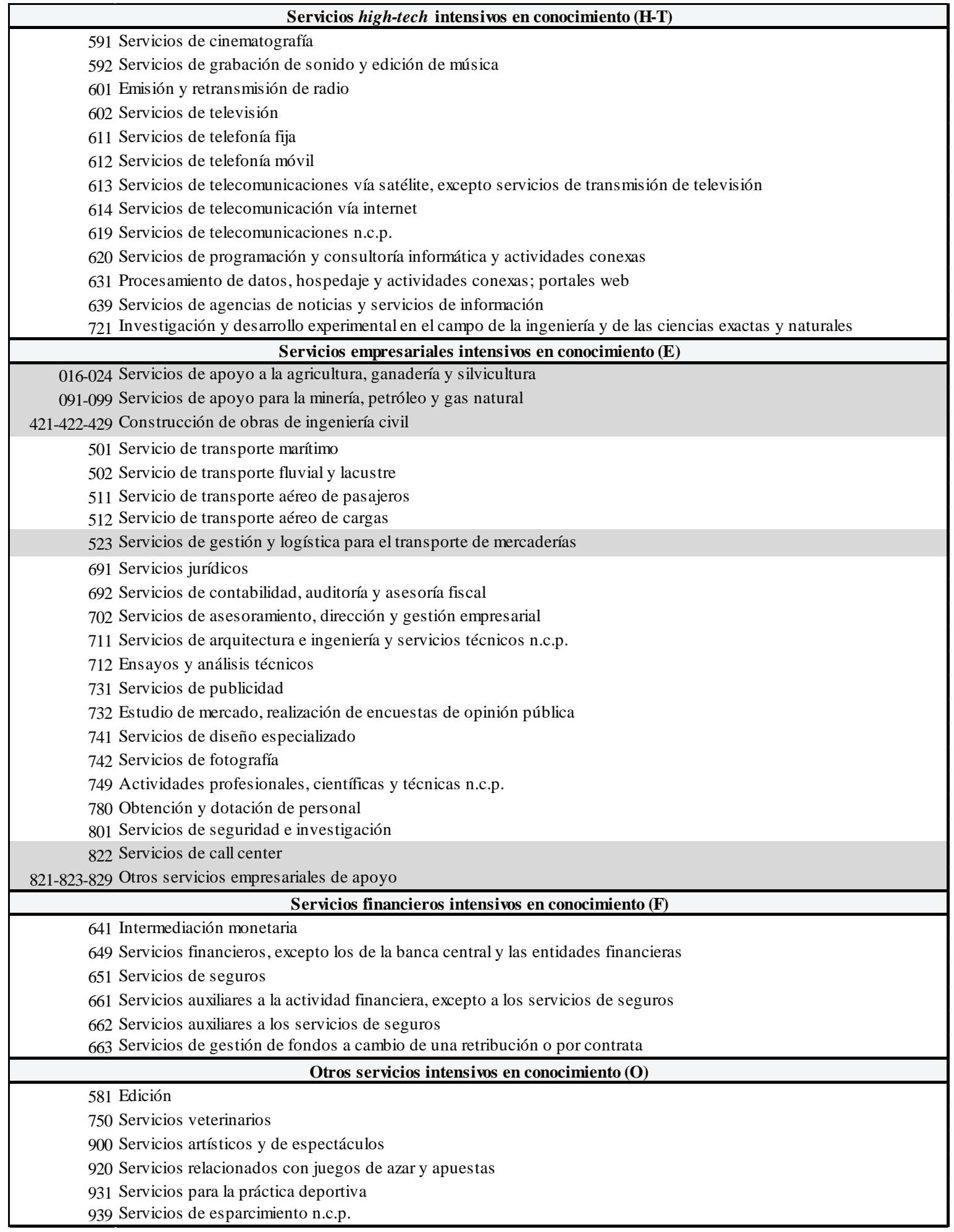

${ }^{2}$ La Clasificación de Actividades Económicas de la AFIP (CLAE-AFIP) se basa en la Clasificación Industrial Internacional Uniforme (CIIU) Rev. 4 y la Clasificación Nacional de Actividades Económicas (ClaNAE 2010) del Instituto Nacional de Estadísticas y Censos (INDEC). Aunque pueden haber algunas diferencias puntuales, a los fines del recorte de actividades de este trabajo las clasificaciones NACE y CLAE-AFIP resultan muy similares. 
Sin perder de vista estas limitaciones, consideramos de todas formas que los datos disponibles representan una fuente de información valiosa en un área donde las estadísticas no abundan. Las distintas variables que se utilizan en el análisis multivariado (cuadro 3) abarcan diferentes indicadores tradicionales del desempeño empresarial, que describen aspectos económicos, productivos y/o financieros de las firmas de cada sector.

Cuadro 3. Variables empleadas en el análisis

\begin{tabular}{|c|c|c|c|}
\hline Variable & Descripción & $\begin{array}{l}\text { Unidad de } \\
\text { medición }\end{array}$ & $\begin{array}{l}\text { Origen de la } \\
\text { información }\end{array}$ \\
\hline Ventas & $\begin{array}{l}\text { Volumen de ventas promedio: ventas } \\
\text { totales, dividido por el número de } \\
\text { presentaciones }\end{array}$ & $\begin{array}{c}\text { Pesos } \\
\text { corrientes }\end{array}$ & IVA \\
\hline Empleo & $\begin{array}{l}\text { Empleo promedio: cantidad de } \\
\text { empleados, dividido por el número de } \\
\text { empleadores del sector }\end{array}$ & $\begin{array}{l}\text { Cantidad de } \\
\text { empleados }\end{array}$ & $\begin{array}{l}\text { Seguridad } \\
\text { social }\end{array}$ \\
\hline Remuneraciones & $\begin{array}{l}\text { Remuneración promedio para el } \\
\text { sector }\end{array}$ & $\begin{array}{c}\text { Pesos } \\
\text { corrientes } \\
\end{array}$ & $\begin{array}{c}\text { Seguridad } \\
\text { social }\end{array}$ \\
\hline VA_Empleado & $\begin{array}{l}\text { Valor agregado, dividido por el } \\
\text { número de empleados }\end{array}$ & $\begin{array}{c}\text { Pesos } \\
\text { corrientes }\end{array}$ & $\begin{array}{l}\text { Seguridad } \\
\text { social y } \\
\text { Ganancias } \\
\end{array}$ \\
\hline VA_Ventas & $\begin{array}{l}\text { Valor agregado, dividido por las } \\
\text { ventas totales (por 100) }\end{array}$ & Porcentaje & Ganancias \\
\hline Compras_Costo & $\begin{array}{l}\text { Compras netas, dividido por el costo } \\
\text { total (por 100) }\end{array}$ & Porcentaje & Ganancias \\
\hline SalariosProd_Costo & $\begin{array}{l}\text { Salarios de personal de producción, } \\
\text { dividido por el costo total (por 100) }\end{array}$ & Porcentaje & Ganancias \\
\hline SalariosTot_Costo & $\begin{array}{l}\text { Salarios totales (de personal de } \\
\text { producción y operativos), dividido } \\
\text { por el costo total (por 100) }\end{array}$ & Porcentaje & Ganancias \\
\hline BienesUso_Ventas & $\begin{array}{l}\text { Bienes de uso, dividido por las } \\
\text { ventas totales (por 100) }\end{array}$ & Razón & Ganancias \\
\hline BienesUso_Activo & $\begin{array}{l}\text { Bienes de uso, dividido por los } \\
\text { activos totales (por 100) }\end{array}$ & Porcentaje & Ganancias \\
\hline ResultadoB_Activo & $\begin{array}{l}\text { Resultado bruto (utilidades menos } \\
\text { pérdidas), dividido por los activos } \\
\text { totales (por 100) }\end{array}$ & Razón & Ganancias \\
\hline VentasN_Activo & $\begin{array}{l}\text { Ventas netas, dividido por los } \\
\text { activos totales (por 100) }\end{array}$ & Razón & Ganancias \\
\hline
\end{tabular}

Estos datos serán empleados para realizar, primero, un análisis de componentes principales y, en segunda instancia, un análisis cluster a partir de los componentes previamente extraídos. Esto nos ha llevado a que algunos otros indicadores alternativos disponibles tuvieran que ser desechados, ya que no cumplían con los requisitos estadísticos para formar parte del análisis de componentes principales: análisis de la matriz de correlaciones, test de esfericidad de Bartlett, medida de adecuación muestral KMO, análisis de las comunalidades (para más detalles, ver Hair, Black, Babin y Anderson, 2010).

En términos generales, el propósito central del análisis factorial, y del método de componentes principales en particular, es poder definir y comprender la estructura subyacente a las variables analizadas; o en otras palabras, condensar la mayor parte de la información y variabilidad contenida en un conjunto de variables originales a partir de un menor número de dimensiones comunes, que en última instancia responden a combinaciones lineales de aquellas variables (Hair et al., 2010; Johnson y Wichern, 2008). Esto nos es de particular utilidad, puesto que permite reunir la información compartida por declaraciones impositivas diferentes y que, por ende, cubren a distintos universos de empresas en cada sector.

Por otra parte, el análisis factorial puede funcionar más como un medio para un fin que como un fin en sí mismo (Johnson y Wichern, 2008), ya que dado que los factores o componentes principales no se encuentran correlacionados entre sí podrían aprovecharse como insumos intermedios para otras técnicas como, por ejemplo, el análisis cluster. Por último, los supuestos y criterios necesarios para una correcta aplicación del análisis de factores pueden ser de utilidad al momento de seleccionar (las mejores) variables entre un conjunto más amplio de indicadores. 
Un punto a tener en cuenta es que la técnica de componentes principales es sensible a cambios de escala o al uso de diferentes rangos de medidas, por lo que inicialmente es necesario estandarizar las variables, algo que por lo general la mayoría de los programas estadísticos suele realizar por medio de los $Z$ scores. Es decir, a las variables originales se les resta la media y luego se dividen por el desvío estándar, de forma que una vez estandarizadas tengan media 0 y desvío 1.

Por su parte, la técnica de análisis cluster o de conglomerados comparte con el análisis factorial el propósito de evaluar la estructura subyacente a los datos. No obstante, mientras que el análisis de factores suele emplearse para agrupar variables en función de los patrones de correlación existentes, la conformación de clusters apunta a generar grupos de casos sobre la base de la proximidad entre los mismos (Hair et al., 2010). El objetivo ulterior del análisis cluster es maximizar la homogeneidad (en función de las características seleccionadas) entre los casos incluidos dentro de cada cluster, al mismo tiempo que se maximiza la heterogeneidad entre los clusters conformados. Esto permite distinguir las particularidades de cada grupo, asignarles un nombre o descripción a cada uno y, en función de ello, definir una tipología empírica.

Entre los métodos disponibles para definir la conformación de los clusters, en este trabajo recurrimos a la técnica no jerárquica de KMedias (el método no jerárquico más común, por lo que en algunos programas estadísticos es el único que figura) y a la distancia Euclídea como medida de proximidad (la más usual para esta técnica, al punto que en ciertos programas viene así por defecto). A diferencia de la irreversibilidad de los métodos jerárquicos ${ }^{2}$, dado que una vez que dos casos o clusters se unieron, quizás de manera incorrecta, no hay forma de que los mismos puedan re-localizarse correctamente en etapas posteriores (Hair et al., 2010; Johnson y Wichern, 2008), el procedimiento de K-Medias permite una mejor asignación de los casos entre los diferentes clusters. Mediante una serie de iteraciones se va redefiniendo la ubicación de cada caso hacia el cluster con media más similar, hasta el punto donde se alcanza la mejor solución posible y nuevas relocalizaciones carecen de sentido. Tal vez el mayor requisito para la aplicación de esta técnica es que debe definirse de antemano el número de clusters a conformar, para lo cual tomaremos como criterio (o stopping rule) a la regla de Calinski-Harabasz $(\mathrm{CH})$. Lamentablemente, no se puede aplicar la lower bound technique, desarrollada especialmente para K-Medias por Steinley y Brusco (2011), ya que, como veremos luego, el número de clusters que se desea verificar excede a la cantidad de variables empleadas (los componentes principales). En casos como este, los mismos autores recomiendan el uso de $\mathrm{CH}$.

\section{Resultados}

\subsection{Principales componentes y dimensiones de análisis}

El primer paso de la aplicación del análisis de componentes principales, a partir de las variables antes señaladas, consiste en definir el número de componentes a extraer. En este caso, el criterio de Kaiser (o del autovalor) sugiere seleccionar los primeros cinco componentes, los cuales en conjunto dan cuenta de más del $80 \%$ de la varianza total (cuadro 4).

Cuadro 4. Componentes, autovalores y varianza explicada

\begin{tabular}{|c|c|c|c|}
\hline Componente & Autovalor & \% Varianza & \% Acumulado \\
\hline 1 & 3,278 & 25,22 & 25,22 \\
\hline 2 & 2,584 & 19,87 & 45,09 \\
\hline 3 & 2,177 & 16,74 & 61,83 \\
\hline 4 & 1,724 & 13,26 & 75,10 \\
\hline 5 & 1,141 & 8,78 & $\mathbf{8 3 , 8 8}$ \\
\hline
\end{tabular}

En segundo lugar, en el cuadro 5 se presentan las cargas factoriales de las distintas variables en los cinco componentes extraídos (luego de aplicar la rotación VARIMAX), lo que ayuda a entender qué tipo de información estarían condensando cada una de estas dimensiones. El primero de los componentes, aquel que da cuenta de la mayor variabilidad (una cuarta parte de la varianza total), presenta un alto peso de los indicadores salariales, como así también cierta incidencia negativa de las compras en el costo total. Por esto, consideramos que este componente representaría al grado de intensidad en recursos humanos. En el caso del segundo componente, sobresalen tanto el resultado bruto como las ventas netas en relación con los activos de las firmas del sector, por lo que podemos sugerir que esta dimensión responde al grado de eficiencia y/o rentabilidad empresarial. En tercer lugar, se destacan el empleo y las ventas promedio, dos variables que reflejarían, cada una desde distintas ópticas, el tamaño y volumen de negocios. El cuarto componente, en tanto, reúne a los dos indicadores alternativos que caracterizan a la intensidad en bienes de uso. Por último, las variables con mayores cargas en el quinto componente son las que representan al valor agregado, las remuneraciones promedio y, nuevamente con signo negativo, la proporción del costo total asignado a compras. Lo interesante de esta dimensión es que el valor agregado en los sectores de servicios intensivos en conocimiento pareciera ir de la mano positivamente del nivel de remuneraciones del personal. En otras palabras, el vínculo entre altas remuneraciones y valor agregado (denominación que asignamos al último componente) daría cuenta nuevamente de la centralidad de los recursos humanos (calificados) en los SIC, pero también podría reflejar, como veremos más adelante, la existencia de ciertas condiciones que permiten en algunos sectores la emergencia de ingresos empresarios extraordinarios que pueden ser parcialmente absorbidos por la fuerza de trabajo respectiva.

\footnotetext{
${ }^{3}$ Los métodos jerárquicos consisten en una serie de pasos de combinación de casos según el grado de similitud (o distancia) entre los mismos, que gráficamente adoptan la forma de un diagrama de árbol o, más técnicamente, un dendrograma.
} 
Cuadro 5. Pesos factoriales y comunalidades de las variables

\begin{tabular}{|l|c|c|c|c|c|c|}
\hline & Comp.1 & Comp.2 & Comp.3 & Comp.4 & Comp.5 & Comunalidad \\
\hline Compras_Costo & $-0,6172$ & 0,0231 & 0,0541 & 0,0726 & $-0,6385$ & 0,7974 \\
\hline SalariosProd_Costo & 0,9521 & 0,1301 & $-0,0438$ & $-0,0748$ & $-0,1092$ & 0,9428 \\
\hline SalariosTot_Costo & 0,9459 & $-0,0164$ & 0,0949 & $-0,1120$ & $-0,1104$ & 0,9288 \\
\hline ResultadoB_Activo & 0,0376 & 0,9742 & $-0,1083$ & $-0,0565$ & 0,0188 & 0,9658 \\
\hline VentasN_Activo & 0,0402 & 0,9743 & $-0,1084$ & $-0,0565$ & 0,0228 & 0,9664 \\
\hline Empleo & 0,2936 & $-0,1746$ & 0,8948 & $-0,0938$ & 0,0287 & 0,9270 \\
\hline Ventas & $-0,2113$ & $-0,1095$ & 0,9097 & $-0,0349$ & 0,2064 & 0,9281 \\
\hline BienesUso_Ventas & $-0,0782$ & $-0,2341$ & $-0,0482$ & 0,9303 & 0,1117 & 0,9413 \\
\hline BienesUso_Activo & $-0,1084$ & 0,1046 & $-0,0755$ & 0,9260 & $-0,0318$ & 0,8868 \\
\hline VA_Empleados & $-0,3083$ & 0,2673 & 0,3506 & $-0,0496$ & 0,6253 & 0,6828 \\
\hline VA_Ventas & $-0,2283$ & 0,0984 & 0,1888 & 0,3024 & 0,7574 & 0,7625 \\
\hline Remuneraciones & $-0,1402$ & $-0,4498$ & 0,2144 & $-0,0725$ & 0,6146 & 0,6509 \\
\hline
\end{tabular}

\subsection{Clusters y tipos de SIC}

Sobre la base de los cinco componentes estimados para cada uno de los sectores de SIC, realizamos luego un análisis cluster no jerárquico. En el siguiente cuadro pueden apreciarse los valores arrojados por el test de $\mathrm{CH}$ si se conforman entre 2 y 9 clusters, donde se resaltan (en negrita) los números recomendados según esta regla. Usualmente, la aglutinación de casos muy diversos entre sí en una cantidad acotada de conglomerados puede no ser la opción más adecuada, por lo que un menor número de clusters suele ser descartado. En el otro extremo, mientras más clusters se formen, mejor se podrán reflejar las diferencias entre casos, incluso pudiendo conformarse uno o varios clusters de un único miembro (en el límite, podrían existir tantos clusters como casos). Sin embargo, no es esto lo que se busca precisamente con los ejercicios de clusterización, por lo que es recomendable además seguir algún criterio de parsimonia que apunte a adoptar la menor cantidad de clusters recomendada por la regla (Hair et al., 2010). En este sentido, optamos por un número de seis clusters para la aplicación del método de K-Medias.

Cuadro 6. Stopping rule para K-Medias

\begin{tabular}{|c|c|c|c|c|c|c|c|c|}
\hline & \multicolumn{7}{|c|}{ Número de clusters } \\
\cline { 2 - 9 } & 2 & 3 & 4 & 5 & $\mathbf{6}$ & 7 & $\mathbf{8}$ & $\mathbf{9}$ \\
\hline Calinski/Harabasz ps eudo-F & 6,60 & 9,20 & 10,90 & 10,31 & $\mathbf{1 1 , 3 1}$ & 9,61 & $\mathbf{1 4 , 4 2}$ & $\mathbf{1 3 , 3 4}$ \\
\hline
\end{tabular}

Otro tipo de validación consiste en verificar que los seis clusters que se conforman a partir de la técnica de K-Medias efectivamente exhiben características heterogéneas. Como puede apreciarse de forma satisfactoria en el cuadro 7 , el análisis de la varianza
(ANOVA) sostiene que la media de los distintos conglomerados para cada variable de estudio es significativamente diferente entre sí (la hipótesis nula del contraste es que la media de los grupos es igual).

Cuadro 7. Análisis de la varianza (ANOVA). Nivel de significación: ${ }^{*} \mathrm{p}<0,05 ;{ }^{* *} \mathrm{p}<0,01 ;{ }^{* * *} \mathrm{p}<0,001$.

\begin{tabular}{|c|c|c|c|c|}
\hline Componente & $\begin{array}{c}\text { Suma de } \\
\text { cuadrados }\end{array}$ & $\begin{array}{c}\text { Grados de } \\
\text { libertad }\end{array}$ & $\begin{array}{c}\text { Media } \\
\text { cuadrática }\end{array}$ & F \\
\hline 1 & 5,737 & 5 & 0,422 & $13,583 * * *$ \\
\hline 2 & 4,61 & 5 & 0,56 & $8,235 * * *$ \\
\hline 3 & 7,436 & 5 & 0,215 & $34,558 * * *$ \\
\hline 4 & 5,303 & 5 & 0,475 & $11,158 * * *$ \\
\hline 5 & 4,808 & 5 & 0,536 & $8,977 * * *$ \\
\hline
\end{tabular}

En cuanto al análisis de los clusters conformados (gráfico 1), un primer aspecto general a resaltar es que cada uno de estos cinco grupos reúne a sectores de servicios catalogados por Eurostat bajo distintas categorías (SIC High-Tech, Empresariales, Financieros y Otros). También es interesante que cuatro de los cinco clusters contengan a uno o más sectores considerados de alta tecnología junto con ramas de 
actividad provenientes de otros tipos de SIC. Esto refleja la necesidad de validar las categorías teóricas contra el uso de información empírica que refleja las características estructurales de los distintos sectores.

Otra cuestión destacable es que la utilización de datos desagregados a 3 dígitos permite apreciar que varios sectores de servicios, a pesar de provenir de antemano de una misma categoría estadística a 2 dígitos, aparecen aquí posicionados en diferentes clusters. En otras palabras, varios de estos sectores hermanos presentan características significativamente heterogéneas entre sí. Quizás el ejemplo más evidente sea el de los distintos subsectores de telecomunicaciones (rama 61), que aquí aparecen desperdigados en cuatro clusters, pero algo similar ocurre con los servicios jurídicos y contables, financieros y técnicos, entre otros.

Gráfico 1. Sectores de SIC por cluster

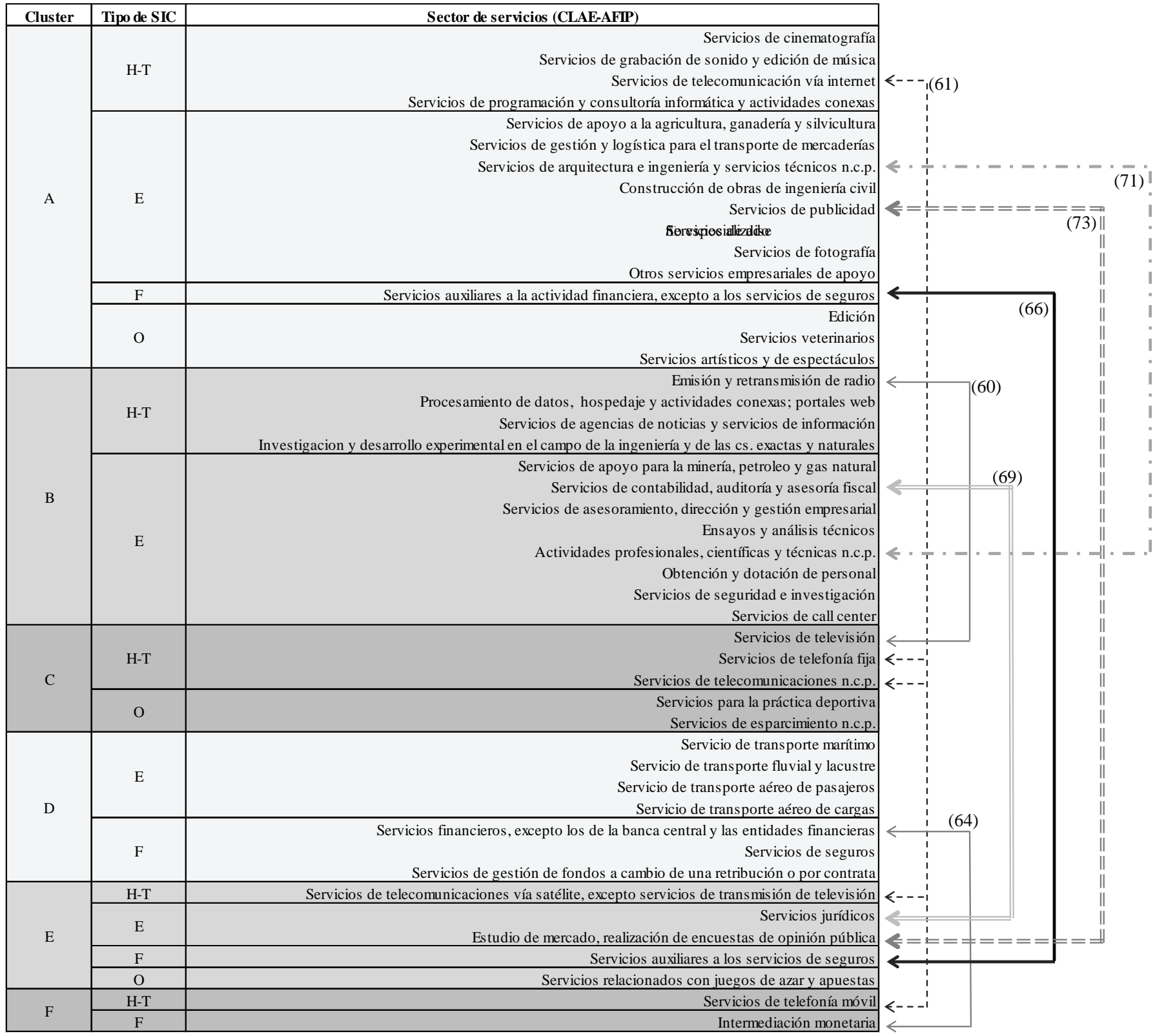

Una forma de entender mejor qué características diferenciales estarían reflejando cada uno de los clusters conformados consiste en analizar las medias de cada conglomerado para las distintas dimensiones consideradas, en este caso, los cinco componentes principales estimados previamente. Como puede apreciarse en el gráfico 2, el cluster A presenta varias dimensiones en torno o levemente por debajo del cero (que, en cierta medida, representa al promedio general). Otra cualidad de este conglomerado es que reúne a ramas de SIC procedentes de las cuatro categorías de Eurostat (desde audiovisuales hasta software, pasando por diversos servicios más especializados). Teniendo en cuenta ambos aspectos, sugerimos denominar a dicho conjunto como el cluster promedio. 
A diferencia del A, el resto de los clusters se distingue positivamente en alguna (o algunas) de las cinco dimensiones de análisis. Por ejemplo, el conglomerado B exhibe la mayor intensidad en recursos humanos de todos los clusters, mientras que en el resto de los componentes principales se encuentra muy próximo al cero. Este conglomerado incluye algunas ramas high-tech en donde se emplea personal altamente especializado (como I+D e ingeniería) pero también otras que se caracterizan por el empleo intensivo de mano de obra de nivel medio de calificación (e.g. call centers). En una caracterización general, se trata de actividades en donde la expansión o achicamiento de los negocios va muy pegada a movimientos similares en la dotación de personal. Por ello, denominados al cluster B como SIC intensivos en recursos humanos.

En el caso del cluster C, la característica dominante es la alta intensidad en la utilización de bienes de capital. Esta es una particularidad esperable entre ramas de telecomunicaciones o televisión, que dependen fuertemente de redes de infraestructura, como así también en el caso de los servicios deportivos y de esparcimiento, donde lo edilicio juega un papel importante. Denominamos a este conjunto como SIC intensivos en bienes de uso.

Por su parte, el cluster D también responde a una lógica clara, ya que aglutina a los distintos servicios de transporte y a buena parte de los servicios financieros, todos sectores en donde, como es conocido, las remuneraciones salariales promedio son elevadas. Esto puede reflejar en ciertos casos la calificación del personal respectivo, pero también las particulares condiciones de negociación laboral en ramas que desarrollan actividades de alto impacto social y económico y están altamente reguladas desde la política pública. A este cluster lo designamos como SIC con alta remuneración al personal.

En el caso del cluster E vuelve a aparecer la particularidad de reunir ramas de todas las categorías definidas por Eurostat. La otra característica sobresaliente de este grupo es la mayor eficiencia y/o rentabilidad con respecto al resto de los conglomerados, que va de la mano de la existencia de altas remuneraciones promedio (no sorprendentemente, los niveles de valor agregado por ventas y empleado son también elevados). Cabe destacar además que, salvo en el caso de la rama estudios de mercado, se trata de sectores sujetos a regulaciones públicas en materia de precios-tarifas y acceso al mercado. Proponemos denominar al cluster $\mathrm{E}$ como SIC de alto valor agregado y rentabilidad. Por último, el cluster F se compone de sólo dos sectores de servicios cuya particularidad principal pasa por el gran volumen de sus operaciones y niveles de empleo: los servicios de telefonía móvil y los servicios bancarios. Se trata de dos casos de SIC de gran tamaño y volumen de actividad.

Gráfico 2. Medias de los clusters en cada dimensión

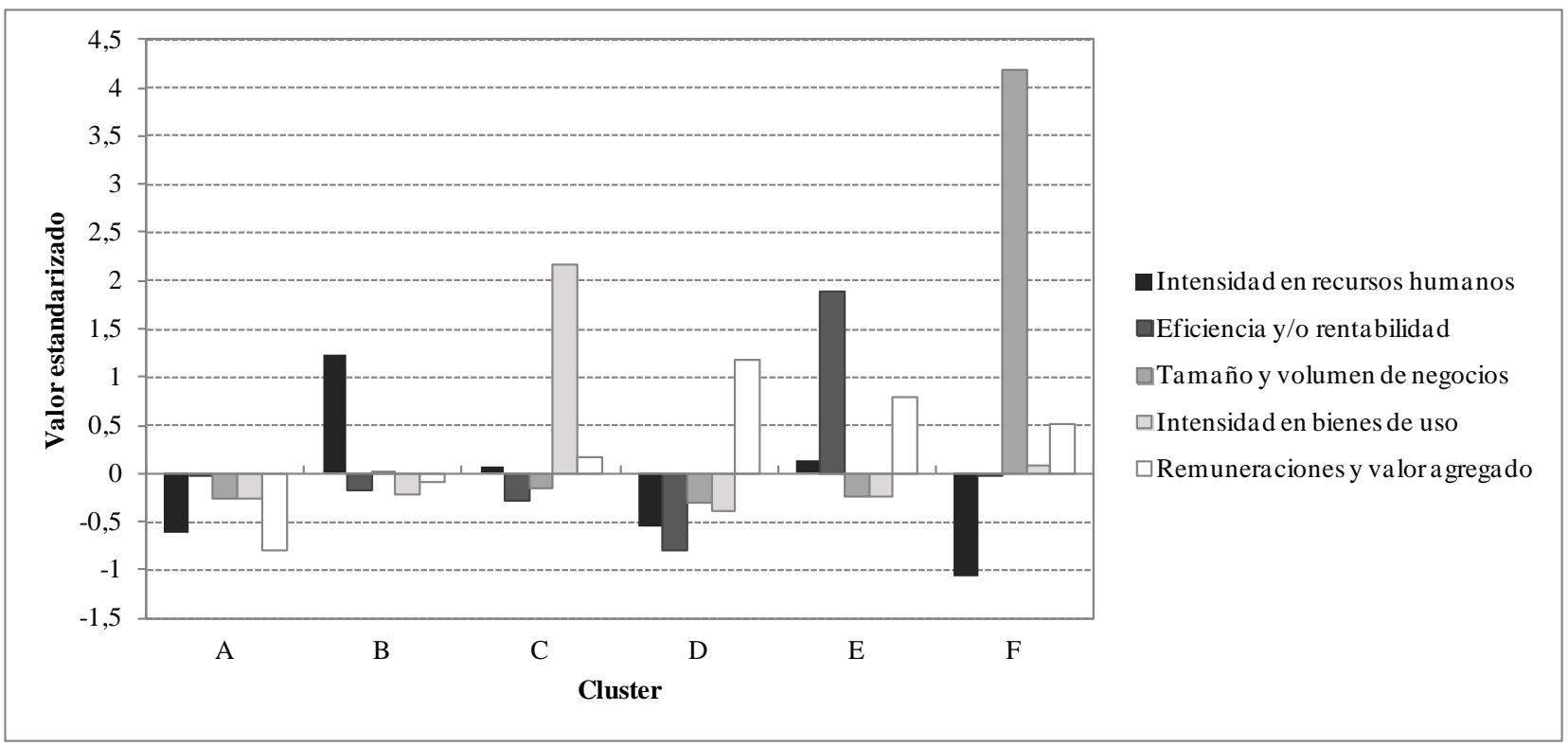

\section{Reflexiones finales}

Al presente no hay dudas de que el sector servicios, tal y como está definido en las clasificaciones estadísticas estándar, reúne a actividades muy diversas entre sí. Sin embargo, la literatura es menos clara acerca de si esto también se refleja hacia el interior de un conjunto de actividades conocidas como servicios intensivos en conocimiento, los cuales han atraído un interés creciente debido al papel que pueden jugar en el desempeño y la productividad del resto de las actividades económicas y en la generación y difusión de nuevo conocimiento.
En la revisión bibliográfica se destacó que algunos trabajos consideran que los SIC tendrían características relativamente homogéneas, mientras que varios aportes empíricos se limitan únicamente a comparar los SIC con el resto de los servicios o, en el mejor de los casos, a dividir a los SIC en algunas categorías agregadas. Los resultados del presente artículo, si bien preliminares y con diversas limitaciones, muestran que al trabajar con datos más desagregados la apariencia de homogeneidad tiende a desaparecer y surgen una serie de características o dimensiones heterogéneas entre los distintos sectores considerados intensivos en conocimiento. Esta contribución empírica se apoya en 
la utilización de datos sectoriales a tres dígitos de desagregación, en contraste con el grueso de la literatura (e incluso de la clasificación de Eurostat) que se basa en información a uno o dos dígitos y, por ende, excluye la posibilidad de identificar diferencias entre los subsectores allí agrupados. Por otro lado, en lugar de trabajar con una tipología teórica definida ex-ante, se buscó abrir la caja negra de los SIC y utilizar técnicas de análisis multivariado para que los diferentes patrones, en caso de existir, surjan ex-post de los propios datos. No son muchos los estudios que hayan realizado un ejercicio de esta naturaleza, y menos aún en el contexto de países en desarrollo como los latinoamericanos.

Dicho esto, cabe destacar, no obstante, que lejos estamos de haber querido presentar una tipología definitiva de los sectores de SIC. Como hemos señalado, en la literatura abundan los criterios y las clasificaciones, lo que en cierta medida responde además a la disponibilidad de diferentes fuentes de información (de distintos países y/o con diversos sectores, variables, etc.) como así también a las metodologías utilizadas. En este caso, si bien la evidencia resulta novedosa para un país en desarrollo como la Argentina, quedan varios aspectos pendientes que pueden dar pie a futuros trabajos. Uno es que al trabajar con promedios sectoriales se pierde de vista la posible heterogeneidad entre las firmas que componen cada sector, por lo cual habría que intentar acceder a información a nivel de empresa. Por otro lado, si bien la información tributaria disponible permite trabajar con indicadores económicos, productivos y/o financieros tradicionales, queda un espacio todavía para aportar a otras discusiones comunes en la literatura como, por ejemplo, si los distintos clusters presentan a su vez patrones de innovación diferentes.

Por último, y de cara al diseño e implementación de políticas para el sector servicios y, más específicamente, para los SIC, este artículo llama la atención respecto de la necesidad de tomar en cuenta la heterogeneidad inherente a las actividades desarrolladas y a las diferentes realidades empresariales en los distintos subsectores. Este paso ayudaría a direccionar mejor los esfuerzos públicos dirigidos a promover estas actividades, siendo que, al presente, los SIC podrían jugar un rol central en los procesos de desarrollo en el mundo emergente.

\section{Referencias}

Aboal, D. y Garda, P. (2016). Technological and non-technological innovation and productivity in services vis-à-vis manufacturing sectors. Economics of Innovation and New Technology, 25(5), pp. 435-454. DOI: $10.1080 / 10438599.2015 .1073478$.

Álvarez, R., Bravo-Ortega, C. y Zahler, A. (2015). Innovation and productivity in services: Evidence from Chile. Emerging Markets Finance and Trade, 51(3), pp. 593-611. DOI: 10.1080/1540496X.2015.1026696

Asikainen, A.-L. (2015). Innovation modes and strategies in knowledge intensive business services. Service Business, 9(1), pp. 77-95. DOI: 10.1007/s11628-013-0219-5.

Bastos, S. Q. A. y Perobelli, F. S. (2012). The search of homogeneity in the heterogeneity: An analysis of the service sector in Brazil. II Conferencia Internacional REDLAS, Santiago de Chile, 18-19 Octubre.
Becker, M., Böttcher, M. y Klingner, S. (2011). Systemising service classifications. International RESER Conference.

Borrastero, C. (2014). Tipología de empresas innovadoras en el sector de software de Argentina según el acceso a las políticas públicas nacionales. Facultad de Ciencias Sociales, Universidad de Buenos Aires.

Browning, H. L. y Singelmann, J. (1975). The emergence of a service society: Demographic and sociological aspects of the sectoral transformation of the labour force in the USA. Austin: Texas University.

Clark, C. (1940). The conditions of economic progress. Londres: Macmillan.

Consoli, D. y Elche-Hortelano, D. (2010). Variety in the knowledge base of Knowledge Intensive Business Services. Research Policy, 39(10), pp. 1303-1310. DOI:10.1016/j.respol.2010.08.005.

Cook, D. P., Goh, C. H. y Chung, C. H. (1999). Service typologies: A state of the art survey. Production and Operations Management, 8(3), pp. 318-338. DOI: 10.1111/j.1937-5956.1999.tb00311.x.

Corrocher, N., Cusmano, L. y Morrison, A. (2009). Modes of innovation in knowledge-intensive business services: Evidence from Lombardy. Journal of Evolutionary Economics, 19(2), pp. 173-196.

Crespi, G., Tacsir, E. y Vargas, F. (2014). Innovation and productivity in services: Empirical evidence from Latin America. IDB Technical Note No. 690. Inter-American Development Bank.

Chica Mejía, J. E. (2011). Definición de los sectores económicos intensivos en conocimiento a partir de la clasificación que hace la OCDE y el análisis del nivel de cualificación de los trabajadores: análisis para Catalunya. Report No. 02. Centre de Política de Sòl i Valoracions, Universitat Politècnica de Catalunya.

David, A. H. (2014). A service sector classification scheme using economic data. The Service Industries Journal, 34(4), pp. 335-353. DOI: 10.1080/02642069.2013.778974.

De Jong, J. y Marsili, O. (2006). The fruit flies of innovations: A taxonomy of innovative small firms. Research Policy, 35(2), pp. 213-229. DOI: 10.1016/j.respol.2005.09.007.

Den Hertog, P. (2000). Knowledge-intensive business services as coproducers of innovation. International Journal of Innovation Management, 4(4), pp. 491-528. DOI: 10.1142/S136391960000024X.

Den Hertog, P. y Bilderbeek, R. (2000). The new knowledge infrastructure: the role of technology-based knowledge-intensive business services in national innovation systems. En M. Boden y I. Miles (Eds.), Services and the knowledge-based economy. Londres: Continuum.

Doloreux, D., Amara, N. y Landry, R. (2008). Mapping regional and sectoral characteristics of Knowledge-Intensive Business Services: Evidence from the Province of Quebec (Canada). Growth and Change, 39(3), pp. 464-496. DOI: 10.1111/j.1468-2257.2008.00434.x. 
Dutrénit, G., De Fuentes, C., Santiago, F., Torres, A. y Gras, N. (2013). Innovation and productivity in the service sector: The case of Mexico. IDB Discussion Paper No. 293. Inter-American Development Bank.

Elche Hortelano, M. D. y Gongález-Moreno, Á. (2007). Innovation in service firms: Exploratory analysis of innovation patterns. Management Research: Journal of the Iberoamerican Academy of Management, 5(2), pp. 113-126. DOI: 10.2753/JMR1536-5433050204.

Evangelista, R. (2000). Sectoral patterns of technological change in services. Economics of Innovation and New Technology, 9(3), pp. 183222. DOI: 10.1080/10438590000000008.

Fisher, A. G. (1939). Production, primary, secondary and tertiary. Economic Record, 15(1), pp. 24-38. DOI: 10.1111/j.1475-4932.1939.tb01015.x.

Freel, M. (2006). Patterns of technological innovation in knowledgeintensive business services. Industry and Innovation, 13(3), pp. 335358. DOI: $10.1080 / 13662710600859157$.

Gallego, J. M., Gutiérrez, H. y Taborda, R. (2015). Innovation and productivity in the Colombian service and manufacturing industries. Emerging Markets Finance and Trade, 51(3), pp. 612-634. DOI: 10.1080/1540496X.2015.1026698.

García-Quevedo, J., Mas-Verdú, F. y Montolio, D. (2013). What types of firms acquire knowledge intensive services and from which suppliers? Technology Analysis \& Strategic Management, 25(4), pp. 473-486. DOI: 10.1080/09537325.2013.774348.

García Manjón, J. V. (2008). Concentración de sectores intensivos en conocimiento y de alta tecnología: El caso de España. Journal of Technology Management \& Innovation, 3(4), pp. 66-79. DOI: 10.4067/ S0718-27242008000200006.

Glückler, J. y Hammer, I. (2011). A pragmatic service typology: Capturing the distinctive dynamics of services in time and space. The Service Industries Journal, 31(6), pp. 941-957. DOI: 10.1080/02642060903078743.

Glückler, J. y Hammer, I. (2013). A new service typology: Geographical diversity and dynamics of the German service economy. En J. R. Cuadrado-Roura (Ed.), Service industries and regions: Growth, location and regional effects. Londres: Springer.

Hair, J., Black, W., Babin, B. y Anderson, R. (2010). Multivariate data analysis. Londres: Pearson.

Hipp, C. y Grupp, H. (2005). Innovation in the service sector: The demand for service-specific innovation measurement concepts and typologies. Research Policy, 34(4), pp. 517-535. DOI: 10.1016/j.respol.2005.03.002.

Hollenstein, H. (2003). Innovation modes in the Swiss service sector: A cluster analysis based on firm-level data. Research Policy, 32(5), pp. 845-863. DOI: 10.1016/S0048-7333(02)00091-4.
Howells, J. (2000). Innovation \& services: New conceptual frameworks. CRIC Discussion Paper No. 38. Centre for Research on Innovation and Competition, The University of Manchester.

Johnson, R. y Wichern, D. (2008). Applied multivariate statistical analysis. Londres: Pearson.

Jordá Borrell, R. M. (2007). Comportamientos innovadores de las empresas de servicios avanzados: Aprendizaje y entorno en Andalucía. Economía Industrial, 363, pp. 205-221.

Lee, K.-R., Shim, S.-W., Jeong, B. S. y Hwang, J.-T. (2003). Knowledge intensive service activities (KISA) in Korea's innovation system. Science \& Technology Policy Institute (STEPI).

Liu, C.-H., Wang, C.-C. y Lee, Y.-H. (2008). Revisit service classification to construct a customer-oriented integrative service model. International Journal of Service Industry Management, 19(5), pp. 639661. DOI: 10.1108/09564230810903505.

López, A. (2018). Los servicios basados en conocimiento: ¿Una oportunidad para la transformación productiva en Argentina?. Documento de Trabajo del IIEP No. 31. Instituto Interdisciplinario de Economía Política de Buenos Aires (IIEP-BAIRES).

López, A., Niembro, A. y Ramos, D. (2014). La competitividad de América Latina en el comercio de servicios basados en el conocimiento. Revista de la CEPAL, 113, pp. 23-41.

Lovelock, C. H. (1983). Classifying services to gain strategic marketing insights. The Journal of Marketing, 47(3), pp. 9-20.

Meiren, T., Edvardsson, B., Jaakkola, E., Khan, I., Reynoso, J., Schäfer, A., ... Witell, L. (2015). Derivation of a service typology and its implications for new service development. The Naples Forum on Service.

Miles, I., Kastrinos, N., Bilderbeek, R., Den Hertog, P., Flanagan, K., Huntink, W. y Bouman, M. (1995). Knowledge-Intensive Business Services: Users, carriers and sources of innovation. European Innovation Monitoring System (EIMS) Report.

Miozzo, M. y Soete, L. (2001). Internationalization of services: A technological perspective. Technological Forecasting and Social Change, 67(2), pp. 159-185. DOI: 10.1016/S0040-1625(00)00091-3.

Muller, E. y Doloreux, D. (2009). What we should know about knowledge-intensive business services. Technology in Society, 31(1), pp. 64-72. DOI: 10.1016/j.techsoc.2008.10.001.

Muller, E. y Zenker, A. (2001). Business services as actors of knowledge transformation: The role of KIBS in regional and national innovation systems. Research Policy, 30(9), pp. 1501-1516. DOI: 10.1016/S0048-7333(01)00164-0.

Pavitt, K. (1984). Sectoral patterns of technical change: Towards a taxonomy and a theory. Research Policy, 13(6), pp. 343-373. DOI: 10.1016/0048-7333(84)90018-0. 
Rubalcaba, L., Gallego, J. y Den Hertog, P. (2010). The case of market and system failures in services innovation. The Service Industries Journal, 30(4), pp. 549-566. DOI: 10.1080/02642060903067571.

Schmenner, R. W. (1986). How can service businesses survive and prosper? Sloan Management Review, 27(3), pp. 21-32.

Schnabl, E. y Zenker, A. (2013). Statistical classification of knowledge-intensive business services (KIBS) with NACE Rev. 2. evoREG Research Note No. 25.

Shafti, F., Van Der Meer, R. y Williams, T. (2007). An empirical approach to service classification for productivity management studies. The Service Industries Journal, 27(6), pp. 709-730. DOI: 10.1080/02642060701453197.

Silvestro, R., Fitzgerald, L., Johnston, R. y Voss, C. (1992). Towards a classification of service processes. International Journal ofServiceIndustry Management, 3(3), pp. 62-75. DOI: 10.1108/09564239210015175.

Steinley, D. y Brusco, M. J. (2011). Choosing the number of clusters in K-means clustering. Psychological Methods, 16(3), pp. 285-297. DOI: $10.1037 / \mathrm{a} 0023346$.

Stigler, G. J. (1956). Trends in employment in the service industries. Princeton: Princeton University Press.

Tello, M. D. (2017). Innovación y productividad en las empresas de servicios y manufactureras: El caso del Perú. Revista de la CEPAL, 121, pp. 73-92.

Tether, B. S. (2003). The sources and aims of innovation in services: Variety between and within sectors. Economics of Innovation and New Technology, 12(6), pp. 481-505.

Tether, B. S. (2005). Do services innovate (differently)? Insights from the European innobarometer survey. Industry \& Innovation, 12(2), pp. 153-184. DOI: 10.1080/1043859022000029221.
Tether, B. S. y Hipp, C. (2002). Knowledge intensive, technical and other services: Patterns of competitiveness and innovation compared. Technology Analysis \& Strategic Management, 14(2), pp. 163-182. DOI: 10.1080/09537320220133848.

Tödtling, F., Lehner, P. y Trippl, M. (2006). Innovation in knowledge intensive industries: The nature and geography of knowledge links. European Planning Studies, 14(8), pp. 1035-1058. DOI: 10.1080/09654310600852365.

Torrecillas, C. y Brandão Fischer, B. (2011). How attractive are innovation systems for knowledge intensive services' FDI?: A regional perspective for Spain. Journal of Technology Management \& Innovation, 6(4), pp. 45-59. DOI: 10.4067/S0718-27242011000400004.

Verma, R. y Boyer, K. (2000). Service classification and management challenges. Journal of Business Strategies, 17(1), pp. 5-24.

Viitamo, E. (2007). Productivity of business services: Towards a new taxonomy. Research Report No. 188. Lappeenranta University of Technology, Faculty of Technology Management, Department of Industrial Management.

Von Nordenflycht, A. (2010). What is a professional service firm? Toward a theory and taxonomy of knowledge-intensive firms. Academy of Management Review, 35(1), pp. 155-174. DOI: 10.5465/amr.35.1.zok155.

Wong, P. K. y He, Z.-L. (2005). A comparative study of innovation behaviour in Singapore's KIBS and manufacturing firms. The Service Industries Journal, 25(1), pp. 23-42. DOI: 10.1080/0264206042000302397.

Wood, P. (2002). Knowledge-intensive services and urban innovativeness. Urban Studies, 39(5-6), pp. 993-1002. DOI: $10.1080 / 00420980220128417$.

Zenker, A. y Doloreux, D. (2008). KIBS, perceptions and innovation patterns. International Journal of Services Technology and Management, 10(2-4), pp. 337-342. DOI: 10.1504/IJSTM.2008.022127. 
